\title{
The Past Is so Present: Understanding COVID-19 Vaccine Hesitancy Among African American Adults Using Qualitative Data
}

\author{
Wilson Majee ${ }^{1,2}$ (D) Adaobi Anakwe ${ }^{1} \cdot$ Kelechi Onyeaka $^{3} \cdot$ Idethia S. Harvey $^{1}$
}

Received: 8 November 2021 / Revised: 5 January 2022 / Accepted: 10 January 2022 / Published online: 19 February 2022

(c) W. Montague Cobb-NMA Health Institute 2022

\begin{abstract}
Background African Americans (AAs) are disproportionately affected by structural and social determinants of health, resulting in greater risks of exposure to and deaths from COVID-19. Structural and social determinants of health feed vaccine hesitancy and worsen health disparities.

Objective The present study aims to explore vaccine attitudes and intentions among program participants, understand the role of an African American faith-based wellness program in COVID-19 awareness and vaccine uptake, and solicit potential solutions for this deep-rooted public health problem.

Methods Data were collected through 21 in-depth interviews among individuals involved within a community-based wellness program. Sixteen phone and five in-person interviews were conducted with church leaders, lifestyle coaches, and program participants. All interviews were audio-recorded, transcribed verbatim, and inductively and thematically analyzed by three researchers.

Findings Live Well by Faith (LWBF) acted as a trusted information source for COVID-19 resources for the AA community. Services provided by Live Well by Faith included enrolling community members for vaccines, negotiating vaccine provision to and facilitating the establishment of vaccine clinics at AA churches, and connecting community members to healthcare providers. Despite the role Live Well by Faith played, VH was a significant concern due, in part, to historical mistrust of government and pharmaceutical companies conducting unethical healthcare research among Black populations. Other factors included uncertainty about vaccination (vaccines' safety, efficacy, and necessity), social media misinformation, and political affiliation. Participants expressed the need for government to commit resources towards addressing historical factors and building trust with minority populations.

Conclusion Resource targeting programs such as Live Well by Faith that engage faith and community leaders in co-designed shared and culturally grounded interventions can help restore and strengthen trust in vaccines and governments and reduce vaccine hesitancy.
\end{abstract}

Keywords COVID-19 · Vaccine hesitancy $\cdot$ Social determinants of health $\cdot$ Health disparities $\cdot$ Live Well by Faith $\cdot$ African American

\section{Introduction}

Wilson Majee

majeew@missouri.edu

1 Department of Health Sciences, University of Missouri, Columbia, MO, USA

2 Department of Occupational Therapy, Faculty of Community \& Health Science, University of the Western Cape, Cape Town, South Africa

3 Masters of Public Health Program, University of Missouri, Columbia, MO, USA
Despite the scientific breakthrough in developing and distributing safe and effective SARS-CoV-2 (COVID-19) vaccine in the United States (US), vaccine uptake has remained low along the racial divide. African Americans consistently lagged in access to, and COVID-19 vaccinations uptake compared to White Americans. As of November 30, 2021, race/ethnicity for $70 \%$ of people who had received at least one dose of the vaccine was known. Among this population, $58 \%$ were White, $19 \%$ were Hispanic, $10 \%$ were African Americans, $6 \%$ were Asian, $6 \%$ were multiple races, and the 
remainder were American Indian/Alaska Native, or Native Hawaiian/Other Pacific Islander [1]. African Americans also make up a smaller share of people who have recently received at least one dose of the vaccine $(10 \%)$ relative to their share of the population (12\%), while this gap has closed for Hispanic populations [1]. Complex, inter-related causes of such disparities have been suggested: concerns about side effects and the long-term effects on health, medical mistrust, negative attitudes towards vaccines, lack of trust in government, health misinformation, contemporary and historic contextual factors (e.g., the cumulative impact of systemic racism and discrimination), and cultural and religious beliefs [2-10].

Since the pandemic onset, racial and ethnic minorities have had disproportionately higher infection and mortality rates [10-12]. In the US, COVID-19-related deaths per 100000 persons were two times higher among minorities (African Americans, Hispanics, Native Americans) versus White individuals where African Americans constitute only $14.2 \%$ of the total population and Latinos accounted for 18.7\% [6, 13-15]. However, African Americans and Hispanics received smaller shares of vaccinations than their burden of the disease (i.e., number of cases relative to their total population) in most states [16].

Although efforts continue to be made to close this gap in vaccine distribution and uptake, little is being done to address the underlying causes of vaccine hesitancy (VH). Vaccine access occurred in the intersection of racial/ethnic belonging and socioeconomic status (SES), with those at the lower end of the SES spectrum at greater risk of poor health from the virus and reduced access to vaccines $[4,17]$. Ethnic minority groups remain at increased risk of infection and death from COVID-19 as they tend to live and work in environments (e.g., overcrowded housing, workplaces, and transportation) in which COVID-19 is more prone to spread. Nevertheless, studies show that these populations hesitate to receive the COVID-19 vaccine. We need to understand and address VH drivers to attain high vaccination rates across individuals and communities. With the emergence and spread of more aggressive COVID-19 variants (e.g., Delta and Omicron), if vaccine uptake remains at the current low rates among AA, COVID-19 will widen existing disparities in infection and death rates.

\section{Vaccine Hesitancy}

Despite the rapid development of COVID-19 vaccines, their widespread acceptance is still a concern. Vaccine hesitancy, the delay in acceptance, reluctance, or refusal of vaccination despite the availability of vaccination services $[18,19]$, is lurking as a significant barrier to achieving herd immunity. Contemporary research categorizes vaccine hesitancy on a spectrum of acceptant, hesitant, and resistant [20,21], where being hesitant refers to a state of uncertainty about getting a vaccine, while resistant refers to those antivaccine or who object to vaccines. For instance, as of April 2021, 13\% of adult Americans decided they would not be vaccinated, $15 \%$ took a "wait and see" approach, and $6 \%$ would get vaccinated if mandated [22]. This complex behavior is influenced by confidence (trust in the effectiveness and safety of vaccine or healthcare provider/system), complacency (perceived risk of infection and disease or value of the vaccine), convenience (access and barriers to vaccination sites), collective responsibility (willingness to protect others by getting the vaccination shot), and the rising power of the internet $[8$, 19, 23-28].

Vaccine hesitancy is contextual, varying for specific vaccines, places, and times. Disparities in adult COVID-19 vaccination uptake were noted by geographic location [lower in rural counties (38.9\%) than in urban counties (45.7\%)], gender and location [women (41.7\% rural, $48.4 \%$ urban) and men (35.3\% rural, $41.9 \%$ urban] [16], race (34\% Black, $22 \%$ White), political affiliation (Democrat 16\%, Republican $29 \%$, Independent $25 \%$ ), and by age, with younger adults ages 18 to 29 more likely to be resistant [29, 30]. Similarly, in Missouri, although $45.4 \%$ of men compared to $52.5 \%$ of women were fully vaccinated as of October 22, 2021, African Americans in the state had lower vaccination rates (32.4\%) than Asians (50.3\%) and White people (38.9\%) [31]. Lower COVID-19 vaccination rates have the potential of derailing current vaccination efforts and limiting the nation and world's economic recovery. Narrowing this VH spectrum is key to halting the spread of the virus and minimizing the threat from new variants such as Delta and Omicron. Consequently, there is a need for a better understanding of the drivers of $\mathrm{VH}$ to target individuals who are uncertain about their intent to vaccinate, with public health interventions that encourage vaccination [32].

Research suggests that AAs and other minority populations were more hesitant about taking the COVID-19 vaccine than their non-Hispanic White counterparts [33, 34]. Saluja et al. [34] attributed this greater prevalence of VH in Black communities (Black $=36.1 \%$ vs. White $=22.1 \%$, $p=0.03$ ) to government mistrust and the need to wait and observe vaccine side effects before receiving the vaccine. In their cross-sectional analysis of data collected from a predominantly AA sample, Moore and colleagues [20] reported age and housing insecurity as predictors of vaccine hesitancy. AAs who became housing insecure due to the pandemic and those younger than 30 years were more resistant to receive the COVID vaccine. Similarly, Sharma, Barta, and Barta [35] examined factors that contributed to vaccine hesitancy among a sample of 428 unvaccinated AAs; they found that $48 \%$ of AAs were vaccine-hesitant. The vaccine-hesitant participants were younger ( $<41$ years), had Republican 
Table 1 Matrix of Determinants of Vaccine Hesitancy

\begin{tabular}{|c|c|c|}
\hline Matrix group & Matrix factors & Emergent themes \\
\hline Contextual influences & $\begin{array}{l}\text { 1. Communication \& media environment } \\
\text { 2. Influential leaders, immune } \\
\text { 3. Historical influences } \\
\text { 4. Religion/culture/gender/socioeconomic } \\
\text { 5. Politics/policies } \\
\text { 6. Geographical barriers } \\
\text { 7. Perception of pharmaceutical industry }\end{array}$ & $\begin{array}{l}\text { (i) Communication \& media environment } \\
\text { (ii) Historical influences } \\
\text { (iii) Politics } \\
\text { (iv) Perception of pharmaceutical industry } \\
\text { (v) Technological and access barriers }\end{array}$ \\
\hline Individual and group influences & $\begin{array}{l}\text { 8. Personal, family, and/or community member experience } \\
\text { with vaccination, including pain } \\
\text { 9. Beliefs, attitudes } \\
\text { 10. Knowledge/awareness } \\
\text { 11. Health system and providers-trust and } \\
\text { personal experience } \\
\text { 12. Risk/benefits (perceived, heuristics) } \\
\text { 13. Immunization as a social norm }\end{array}$ & $\begin{array}{l}\text { (vi) Personal, family, and/or community } \\
\text { member experience } \\
\text { (vii) Beliefs, attitudes } \\
\text { (viii) Knowledge/awareness } \\
\text { (ix) Perception of healthcare providers }\end{array}$ \\
\hline Vaccine/vaccination-specific issues & $\begin{array}{l}\text { 14. Risk/benefits (epidemiological and scientific evidence) } \\
\text { 15. Introduction of a new vaccine } \\
\text { 16. Mode of administration } \\
\text { 17. Design of vaccination program } \\
\text { 18. Reliability and/or source of vaccine } \\
\text { 19. Vaccination schedule } \\
\text { 20. Costs } \\
\text { 21. Strength of recommendation }\end{array}$ & (x) Introduction of a new vaccine \\
\hline
\end{tabular}

political affiliation (22\%), and lived in the Northeast region of the US (26\%). However, hesitancy to vaccines in this population is not a new phenomenon as previous studies showed that Black adults are less likely to receive vaccines such as the seasonal flu vaccine [36]. These challenges to protecting the health of AAs nested in multilevel individual, systemic, and structural factors are nuanced and need to be better understood to elevate minorities and vulnerable communities towards ending health disparities.

\section{Vaccine Hesitancy Determinant Matrix}

The SAGE Working Group on Vaccine Hesitancy Determinants Matrix groups factors that can influence hesitancy into three main categories: contextual, individual and group, and vaccine/vaccination-specific issues [19]. Contextual factors include geographic barriers, socioeconomic influences, historical influences, and media. Examples of factors that fall under individual and group influences included attitudes about health and prevention, knowledge or awareness, health systems and providers, and risk and benefits. Also, vaccine and vaccination factors include the introduction of new vaccines and the reliability of vaccine and vaccination equipment (Table 1). Together, these factors interact to influence decisions positively or negatively on vaccine uptake. We use the Vaccine Hesitancy Determinants Matrix to guide our data analysis as it encompasses other factors suggested in the validated 5-C Vaccine Hesitancy Model [26, 37]. The qualitative approach we adopted allowed for the flexibility to explore the broad scope of predictors of vaccination behavior. The 5-C model classifies these predictors into five groups: confidence, complacency, convenience (or constraints), risk calculation, and collective responsibility [19, $28,37]$, which all fall within one or more of the three broad categories used in the matrix.

The purpose of the study is to examine factors that contribute to vaccine hesitancy among, and vaccine perceptions of, AA church leaders and church members participating in the Live Well by Faith (LWBF) wellness program, understand the role LWBF played in COVID-19 awareness and vaccine uptake, and solicit potential solutions for this deep-rooted public health problem. In asking church leaders and program participants to share their perspectives on vaccines and vaccinations, this study will provide deeper insights into (1) the underlying causes of $\mathrm{VH}$ in African American population, (2) the role of faith-based health and wellness programs in promoting COVID-19 vaccination, and (3) how the knowledge gained can inform the design of context-based culturally sensitive interventions to address vaccine hesitancy. A more robust examination of how faithbased African American communities collectively think about, and practice vaccination is essential in understanding the interfaith and interfamily dynamics that contribute to VH. Triangulating data collection among churches leaders, lifestyle coaches, and program participants allows for new insights into the varied perspectives African Americans have on vaccines and vaccinations and advances the utility of the concept of $\mathrm{VH}$ more effectively than collecting the 
Table 2 Semi-structured interview guide for LWBF program participants

First, let talk about your personal experience with LWBF

1. Please tell me about yourself:

- Probe: your job, how long have you lived in this community, and when did you start participating in LWBF?

2. How did you first hear about LWBF?

3. What made you want to sign up for the program?

4. Think of all the programs you have participated in; can you pick the top three that you found to be more beneficial to you

- Probe: How have you used what you are learning in the program in your daily life? Give examples where possible

5. Thinking of LWBF, tell me about one of the most important things you got out of the program?

6. How has the program or your participation in the program impacted your community?

- Probe: Are there any organizational or community changes due to the program

7. What other changes have you seen in your community as a result of the program?

8. Do you think there was anything missing in the program? What can be done to improve it?

- Probe: What are the strengths of the program? What are the weaknesses of the program?

9. Do you feel you have enough opportunities to use the skills you gained from the program?

- Probe: How can you be supported in utilizing skills/knowledge from the program

We are almost done, just a few more questions on LWBF and COVID-19

10. Tell me how the LWBF program has been impacted by COVID-19? Probe: Your participation and the functioning of the program in general

11. What have you heard about the COVID-19 Vaccine? How did you hear about it?

12. Has your participation in the program helped you in any way to understand COVID-19 better? How?

13. Do you think the vaccine is a good solution to the pandemic?

14. COVID-19 vaccination is ongoing - Where you vaccinated? Do you think most LWBF participants have been or will be vaccinated - why?

How about the broader African American population, why?

15. What challenges did you face, or you think you will face in getting vaccinated? How can these challenges be addressed?

16. Do you have anything else you want to say about your experiences with LWBF, that I did not ask about?

Thank you so much for taking the time to interview!

perspective of one group of participants. Further, engaging religious segments of the population is critical as religious conservatism is associated with poor vaccine knowledge, lack of trust in science, and higher rates of VH [38-40]. However, the government can engage pastors and other religious leaders to facilitate discussion and nurture trusting relations with people of faith, particularly AAs [41].

\section{Methods}

\section{Setting and Participant Selection}

LWBF is an AA faith-based program started in 2016 through a local health department (LHD) in central Missouri. It addresses chronic health conditions among AAs. It has seven programs in three main areas: self-management, physical activity, and nutrition. Since its inception in 2016, participants grew from 60 in 2016 to 148 in 2019. Fifteen lifestyle coaches serve as program coordinators within ten AA churches. For the study, the community's LHD assisted in recruiting participants using purposive and snowball sampling methods [42]. Following ethics approval by the institutional review board at the first author's university, the study used an exploratory design [43] to collect narratives on the impact of LWBF on the program participants' health and well-being. The inclusion criterion was involvement with LWBF as either a program participant, lifestyle coach, or church leader. All participants in the LWBF were from Christian congregations, primarily Methodist and Baptist churches. No incentives were provided to participants.

\section{Data Collection}

Interviews are apt tools to explore under-researched topics and allow participants to provide rich descriptions in realworld contexts [44]. Sixteen phone and five in-person interviews were conducted in April 2021 with lifestyle coaches, church leaders, and program participants. The five in-person interviews were located at the participants' churches. Interview questions were developed by the research team based on the literature (Matrix of Determinants of Vaccine Hesitancy) and study objectives and reviewed by community partners for understandability and cultural relevance/sensitivity. For example, questions were exploratory (see Table 2) to allow for emergent themes that capture vaccination perceptions and behaviors in participants' lived experiences. Questions broadly examined the efficacy of LWBF. 
The first author conducted all interviews, which lasted approximately $35 \mathrm{~min}$ (range 14 to $57 \mathrm{~min}$ ). Data collection continued until saturation was reached [43, 45].

\section{Data Analysis}

All interviews were transcribed verbatim and analyzed using inductive and deductive thematic analysis. The first and third authors listened to each audio-recording following the interview and a second time prior to beginning the inductive coding to identify key phrases or ideas that would be used as deductive codes during the second round of coding. Codes addressing similar thoughts or ideas were merged. Thematic analysis used by the first three authors was guided by existing concepts and ideas on vaccine hesitancy, in this case, the SAGE Working Group on Vaccine Hesitancy Determinants Matrix of vaccine hesitancy. Following independent familiarization with the data set and the SAGE Working Group on Vaccine Hesitancy Determinants Matrix, the three researchers independently coded five transcripts using the 21 items of the SAGE Working Group on Vaccine Hesitancy Determinants Matrix. The coding process was guided but not restricted by the SAGE Working Group on Vaccine Hesitancy Determinants Matrix. During the coding process, inductive codes that were either separate from or expanded the SAGE Working Group on Vaccine Hesitancy Determinants Matrix codes were allocated to information that illustrated a new theme [46]. Using the three $\mathrm{VH}$ dimensions guided the analyses while allowing for emergent complementary subthemes on African American VH. Following the development of the coding scheme, two authors coded all 21 interviews, and discussed all coded transcripts to verify completeness, ensure reliable coding, and resolve areas of disagreement [47]. Transcripts and codes were entered into NVivo for further analysis by one of the researchers. Exemplar quotes were identified to support each theme.

\section{Researchers}

Members of the research team were racially similar but diverse in their ethnic and gender identifications. These four individuals approached this study with scholarly and personal interests in identity and the meaning for building support, trust, and rapport in the lives of members of marginalized groups. In terms of their roles in this study, the first author, a Ph.D. level community health researcher, conducted all the individual interviews, while the third author, an advanced master student at the time, worked directly with the first author in analyzing transcripts and generating themes. The second author, a Ph.D. level public health researcher, served as an independent auditor. The independent auditor's role was to (1) guarantee that multiple perspectives of the data were honored and discussed, and (2) help ensure that analysts' assumptions, expectations, and biases did not unduly influence the findings [48]. The fourth author, a Ph.D. level community-engaged researcher, had experience working with African American communities, was raised as a Baptist, and provided insights that strengthened the study. Researchers were critically self-reflective about their lived experiences and preconceptions on vaccines and vaccinations [49, 50].

\section{Findings}

A total of 21 participants were interviewed and had an average age of 62.8 years. Most participants were Black (95\%), female $(71 \%)$, and had lived in the community for 11 years or more $(81 \%)$ and had been involved with the program for three or more years $(90.4 \%)$. The participants included five church leaders and five LWBF program participants. Eleven participants had the role of being lifestyle coaches and program participants. A detailed demographic profile for these participants is provided in Table 3 .

The themes that emerged from the analysis are presented in two groups. First, we present three major themes within the dimensions of the Matrix of Determinants: contextual influences, individual and group influences, and vaccine and vaccination-specific issues. The fourth theme represented the "role of the LWBF program" in COVID-19 vaccinations.

\section{Contextual Influences}

Evidence suggests that contextual factors continue to influence perceptions and decisions of AAs' attitudes and beliefs regarding vaccines and vaccinations. Although LWBF provides health literacy programming on physical activity, nutrition, and self-management, intergenerational trauma and historical injustices continue to impact African American families. Of the seven subthemes listed under contextual influences in the Matrix of Determinants of Vaccine Hesitancy, five were identified by study participants: historical influences, perception of the pharmaceutical industry, communication and media environment, technological and access barriers, and politics. The first three were most prominent and are discussed below.

\section{Historical Influences}

Most participants expressed a lack of trust in the government concerning their health. African Americans participating in the LWBF program felt unsafe and lacked confidence in government because of historical unethical healthcare practices and research 
Table 3 Demographic characteristics of study participants

\begin{tabular}{|c|c|}
\hline Characteristics & $\begin{array}{l}\text { Study population } \\
n(\%)\end{array}$ \\
\hline Total number of participants & 21 \\
\hline \multicolumn{2}{|l|}{ Participant type } \\
\hline Church leader & $5(23.81)$ \\
\hline Lifestyle coach & $11(52.38)$ \\
\hline Program participant & $5(23.81)$ \\
\hline \multicolumn{2}{|l|}{ Age (years) } \\
\hline $30-40$ & $3(14.29)$ \\
\hline $60 \&$ above & $18(85.71)$ \\
\hline \multicolumn{2}{|l|}{ Race } \\
\hline Black or African American & $20(95.24)$ \\
\hline White or European American & $1(4.76)$ \\
\hline \multicolumn{2}{|l|}{ Sex } \\
\hline Female & $15(71.43)$ \\
\hline Male & $6(28.57)$ \\
\hline \multicolumn{2}{|c|}{ Income (changes in income since Jan 2020) } \\
\hline N/A & $3(14.29)$ \\
\hline Increased & $2(9.52)$ \\
\hline Decreased & $4(19.05)$ \\
\hline Stayed the same & $12(57.14)$ \\
\hline \multicolumn{2}{|l|}{ Employment } \\
\hline Yes & $8(38.1)$ \\
\hline No & $5(23.81)$ \\
\hline Retired & $8(38.1)$ \\
\hline \multicolumn{2}{|l|}{ Number of years in community } \\
\hline $0-5$ & $3(14.29)$ \\
\hline $5-10$ & $1(4.76)$ \\
\hline $11-15$ & $0(0)$ \\
\hline $16-20$ & $2(9.52)$ \\
\hline More than 20 & $15(71.43)$ \\
\hline \multicolumn{2}{|c|}{ Number of years involvement with LWBF } \\
\hline Less than 1 & $1(4.76)$ \\
\hline $1-2$ & $1(4.76)$ \\
\hline $3-4$ & $9(42.86)$ \\
\hline More than 4 & $10(47.62)$ \\
\hline
\end{tabular}

Note: $L W B F$, Live Well by Faith

conducted in their community. Feelings of betrayal still resonate within AA communities, and "part of that [vaccine hesitancy] is distrust in anything that might be coming out from the government" (Church Leader: 210407_002). African Americans "want to make sure [they are] not being experimented on, like the Tuskegee or the syphilis thing" (Lifestyle Coach: 210413_001). Another Lifestyle Coach described how medical mistrust developed because of the historical mistreatment of African Americans within the US medical system:
${ }^{1}$ During the Trail of Tears, the Indians who were forced to move west were given blankets that were, I think it was typhoid or smallpox, but anyway, they were infected blankets. The intent was to kill them before they got to where they were going. The Tuskegee experiments where Black men were injected with syphilis and never treated, they just monitored to see how it gone. We remember that (Lifestyle Coach: 210419_001).

The Tuskegee Syphilis Study in Black men involved 399 men with syphilis and 201 without the disease. In the study, participant informed consent was not obtained. Instead, participants were informed that they were being treated for "bad blood" - a term used locally to refer to several diseases including syphilis. As incentive to participate, men were provided free medical exams, meals, and burial insurance. However, when penicillin, the treatment of choice for syphilis, became widely available, the Black men in the study were not given access to it [51]. Narratives from participants in our study show that memories of the Tuskegee Syphilis Study as a racist experiment that denied AA effective medication for syphilis reverberate with the AA community. Historically, the US medical system has abused minority communities for the advancement of medicine as stated by a church leader,

What White people just don't get, is that there are places where African American people have learned not to be trusted. Not to trust. And we know about the awful things the US government has done with the African American population. Now, people say, "It's a generation ago." But the memory lives strong in the culture (Church Leader: 210407_003).

Mistrust and lack of confidence in vaccines and vaccination stems from the fact that "for too long, too often, African Americans have been used as test subjects. People don't forget that and don't feel comfortable" (Program participant: 210421_003).

Ongoing and persistent injustices against minorities (i.e., police profiling and brutality against AAs), high incarceration rates, and racial residential segregation of AAs have worsened the mistrust in the US sociopolitical climate and government. Expressing feelings shared by many AAs, one study participant said,

Especially as of late with so many Black people being killed by law enforcement, and they're involved. That's

\footnotetext{
1 The Tuskegee Syphilis Study was conducted between 1932 and 1972 by the United States Public Health Services and the Centers for Disease Control and Prevention. The purpose of the study was to observe the effects of untreated syphilis. African American men were not informed of the nature of the study.
} 
part of the government infrastructure ... We've all been slightly jaded. Especially, I know my generation especially. Because I've seen so many people my age say, 'I'm not getting vaccinated. I'll wait until you all get vaccinated, and we'll see what happens with you guys.' But and I think a lot of it is just, it's just we're extremely jaded because we've unfortunately had to witness a lot of bad things happening recently with the man, or whoever we consider to be, you know, trying to attack us. I think we're on a form of defense for everything. And it's pretty unfortunate because not everything is an attack, but I think, even with the vaccine, we see that as some form of an attack (Program participant: 210417_001).

When injustices persist, they create an environment in which, "Government says a good thing, (but) our people have been programmed almost like the Native Americans, to say it's no good" (Church Leader: 210407_002). Moreover, these sentiments on injustices contribute to VH we experience with minority populations. Referring to the recent police brutality (i.e., George Floyd, Ahmaud Arbery, and Breonna Tyler) and drawing a parallel between the terrorist attacks of September 11, 2021, and the unethical practices on AAs, one church leader stated,

Like every once in a while, I see something on Facebook where they show the World Trade Center on fire and the subscript is, 'never forget.' Like we're supposed to keep remembering what happened, and then in ancillary ways, keep blaming people of Middle East descent and eyeing them with suspicion and denying them access to the US. 'Never forget.' Well, nobody has to tell you that. Nobody has to tell the African American community not to forget. These things are remembered across generations. It's just naive for people to say, 'just forget about it. Go on with your life. That's over and done with.' It's not that easy to forget and every once in a while, something happens to make us remember. Like Mr. Floyd gets murdered by the police (Church Leader: 210407_003).

Along the same vein, another participant expressed,

I think even when we attempt to move on, it's not allowed. They continue to show us how much disregard they have for our lives, in so many ways, I mean, George Floyd. They continue to show us that no, it's not in the past. It's not over. 'We still have it out for y'all.' Those kinds of past experiences in our community are the hesitation (Lifestyle Coach: 210418_001).

In expressing such feelings, the participants communicate their fears and mistrust in the government, particularly regarding their health, and how continued racial discrimination and marginalization influence AAs' decisions around their health (e.g., VH).

\section{Perception of the Pharmaceutical Industry}

Distrust in government and continued acts of injustice influence AAs' perceptions of other actors in the healthcare system. Many participants expressed concerns about the pharmaceutical industry's role in the rapid development of the COVID-19 vaccines. Most people were worried about the Johnson \& Johnson's Janssen vaccine, especially following reports that suggested an increased risk of thrombosis with thrombocytopenia syndrome in adult women younger than 50 years old. Most participants acknowledged that there is mistrust with pharmaceutical companies as "experiences in the past where maybe studies have been done and... you don't know whether they are going to vaccinate something for you to live longer or to die tomorrow" (Lifestyle Coach: 210411_001). Another participant asked, "why are they trying to make us get it? Is there something wrong with it?" (Program participant: 210417_001). Participants expressed anger when talking about the Janssen COVID-19 vaccine. A participant stated, "I will never trust Johnson \& Johnson. They can't even get baby products right. If that's all they had, I wouldn't have taken the shot either" (Lifestyle Coach: 210413_001). Another participant mentioned that "Johnson \& Johnson is the one that didn't tell people that there was asbestos in baby powder" (LWBF Lifestyle Coach: 210414_002).

\section{Communication and Media Environment}

Many factors influence vaccine decision-making, including the impact of misinformation about the vaccines' availability, safety, and effectiveness. Social media misinformation is easily accessible through social platforms, which can have real consequences on attitudes and behavior related to the COVID-19 vaccinations. One participant explained this with regard to the information that was shared on social media,

I think Facebook is definitely a negative to African Americans in terms of getting knowledge. When you go to Facebook and people are talking, 'Don't get the vaccine. It's DNA from aliens. It's something that's going to kill us. This is just another way of them experimenting on us.' When you go to Facebook and look how many people on Facebook, young and old, and they hear that over and over again it becomes a process in their head that says, 'Okay, I'm not going to do it because everybody says don't do it.' That's one thing that's hurting us - listening to the negative parts of the anti-vaccine folks (Church Leader: 210407_002). 
In reference to information shared on social media, another participant argued,

Things from the news, Tik Tok, for example. . . It had this African American lady doing before and after pictures. Apparently, she is fine, but she claimed after she took the vaccine, she ended up in a hospital for so many days where her eyes were swollen shut, and she showed pictures of her in bed. Now whether or not that is true, I don't know, but that's getting into Tik Tok (Lifestyle Coach: 210418_002).

Given the diversity of factors that drive VH in different groups and the need for enhanced vaccine confidence communication must come from trusted messengers and be tailored to the community's values, concerns, motivations, and information needs. Also, while informing the public of vaccine-related health risks is commendable for trustbuilding during a pandemic or crisis, communicating such risks should be done in ways that do not instill fear and less confidence in vaccines. The current media coverage on the side effects of the Janssen COVID-19 vaccine impacted how society viewed vaccinations. One participant said,

The last vaccine that just came out, the Johnson \& Johnson, the news gives out information about blood clots, and people are dying, and things of that nature. I think that a lot of times people would rather take the risk of riding it out before they decide to take the chances of getting a blood clot or dying or getting sick from the vaccine (Program Participant: 210421_002).

\section{Individual and Group Influences}

Three subthemes within the individual and group influences category were prominent in explaining $\mathrm{VH}$ among AAs: knowledge/awareness of vaccine; perception of healthcare providers; and personal, family, or community member experience with vaccination.

\section{Knowledge/Awareness of Vaccine}

In a pandemic, people gather as much information as they can from family, friends, healthcare providers, community leaders, the internet, news, and social media. In such cases, distilling information can be a challenge. Study participants expressed frustration with not knowing the truth about the vaccine, which heightened their hesitancy to take the vaccine. One participant said,

Lack of knowledge because we believe the vaccines to be safe, but they [vaccine] are so new. You hear things. I heard yesterday, a doctor talking about how sick everyone's going to be in 6-12 months (Program participant: 210421_003).

In this vein, another participant added,

I have an 83-year-old mother and she refuses to take the shot because she feels they are trying to alter her DNA. And I'm like, Mom, they're not going to put stuff in the little bitty water and little bitty shot to give to you (Lifestyle Coach: 210413_001).

Some lacked understanding of how vaccines work and believed they did not need to be vaccinated because of their excellent health. One participant explained, "She feels like she takes excellent care of her health, and she takes other kinds of supplements. So, she feels like she's protected." In other words, "It's not necessary for her to be vaccinated" (Church Leader: 210407_002). Others trusted information from their loved ones who had better education as pointed out by this participant,

She studied biology and her logic is true. By the time a vaccine is developed, it's going to mutate and the vaccine you're taking is for the vaccine from last year -- or for the virus of last year, not this year (Lifestyle Coach: 210419_001).

\section{Perception of Healthcare Providers}

The quality of care people received from the healthcare providers also informed their vaccine decision-making. Unfortunately, some of the experiences African Americans had during hospital visits resurrected in their feelings of racism, which fostered mistrust with healthcare providers and their services. One participant explained his experience,

I have asthma and I have borderline COPD. I went in barely breathing the first time and they gave me oxygen treatment. They said, 'If you have any other problems come back.' Prior to the emergency visit my wife and I both tested positive for COVID. They knew this, but they sent me back home with my medical condition. That was the first time. Two days later, I'm back at the emergency room. They gave me some medication to take and sent me back home. The third time I went back in, the doctor came in and he already reviewed my record. He said, 'We're not going to make the mistake a third time.' He said, 'You go in the hospital.' And the whole time I was doing that, I was saying to myself.' Had I been a White person would I have been sent home the first time with my medical condition?' My wife is already in the hospi- 
tal. We've already announced we have COVID. Why would you send me home, back and forth, instead of getting me in and getting me the treatment that I need? (Church Leader: 210407_002).

When they visit their healthcare providers, people's interpersonal and institutional experiences build confidence about their providers and the support they receive. Along the same vein, another participant detailed factors causing her to be hesitant about vaccination and said,

We had a member of our church who had a 16-year-old son who was injured, and he was rushed to the hospital, and they were told, 'we don't take Coloreds here.' It was several, like 100 miles, and they would not even transport the child in an ambulance. When you hear those stories and you hear them from different parts of the country, then you do become very hesitant as to, are we going to take this or not? (Lifestyle Coach: 210419_001).

Ongoing discrimination against people of color in the healthcare system fosters mistrust in healthcare providers and the healthcare system, thereby worsening VH.

\section{Personal, Family, and Community Member Experience with Vaccination (Including Pain, Pre-existing Conditions)}

Regarding personal, family, and community member experiences with vaccination, most participants expressed $\mathrm{VH}$ due to uncertainty on the effect of the vaccine on people with pre-existing health conditions such as diabetes, allergies, high blood pressure, and asthma. One participant said,

If you have a pre-existing condition, the likelihood of you doing anything without a doctor's approval, they won't do it. Whether it is diabetes, high blood pressure, asthma, or having allergies to different things there is a good chance you are not going to get them to do anything until their doctor physically tells them it is okay to take that vaccine. A lot of people are just skeptical. They want to see other people take it and see how they turned out (Church Leader: 210408_001).

Another echoed,

They don't want to get vaccinated because the vaccine has not been proven for people that have food allergies. Well, I have food allergies, and I don't want the vaccine because it's not proven for my specific food allergies. (Lifestyle Coach: 210414_002).

\section{Vaccine and Vaccination-Specific Issues}

\section{Introduction of New Vaccine}

Regarding the third category of factors, introducing a new vaccine - rapid pace of vaccine development - was the main reason for vaccine hesitancy. Emerging research has already cited concerns about the safety of vaccines against COVID-19, including the rapid pace of vaccine development, as one of the primary reasons for hesitancy [52], especially among AAs. One participant noted:

Most people are scared because it was so fast paced, but they don't realize it's fast paced because we've already been developing vaccines and stuff technology has moved up a couple of notches ... They want to make sure we're not being used as guinea pigs. . . I was one of the ones that said I'm going to wait six months (Lifestyle Coach: 210413_001).

The rapid development of the COVID-19 vaccine with a lack of community educational outreach before the vaccination roll-out has increased hesitancy among the participants.

Not much because of the lack of research or lack of education, but just being mindful of giving the vaccines a little bit more time before one would go get the vaccine. I think, right now, it's early. It's too early for a lot of people, and some people are very skeptical of this vaccine (Program participant: 210421_002).

In fact, this was a prominent theme regarding vaccine hesitancy as most study participants felt the same way:

A lot of us know, it takes the FDA forever to approve something, so the fact that they did this in a year, scares a lot of people. Like my daughter's a nurse at a local hospital and she still hasn't gotten it (Program participant: 210421_001).

\section{Role of LWBF}

\section{Trusted Source of Vaccine}

Since the onset of the pandemic, LWBF has been involved in educating program participants on how the virus spreads, necessary precautions to take to protect themselves and others, and the importance of getting vaccinated. All participants acknowledged these roles LWBF played, particularly in providing information on the virus and advocating for vaccination clinics at Black churches. According to participants, LWBF improved access and information dissemination on COVID-19 vaccinations. Participants cited that 
their involvement with LWBF aided in vaccine information access. One participant said, “a lot of people weren't going to get their vaccine until LWBF started promoting it. I think it's helping the Black community" (Program participant: 210414_003). Another said,

I think the majority of people would get vaccinated because of their affiliation with LWBF. As I said before, there is information that is out there that you can find out about (Church Leader: 210408_001).

As discussed under the three themes above, African Americans lack trust in government, healthcare providers, and vaccine development processes and therefore experience high levels of uncertainty and lack of intent to vaccinate against COVID-19. However, participants reiterated how LWBF helped increase vaccine awareness in the AA community by refuting myths surrounding vaccines, as one echoed that:

We have increased knowledge of it and actually I tell them, I' $m$ an example. 'Do you see a horn growing out of my head? I think I'm fine.' If you're worried about the vaccine, pay attention to the results (Lifestyle Coach: 210414_001).

\section{Discussion}

Since the first diagnosed case of COVID-19 in December 2019, and the approval of COVID-19 vaccines in December 2020 , the number of fatalities continued to rise amid slow vaccine uptake. More recently, and following mandatory vaccination orders in many workplaces, COVID-19-related deaths have gone down. Although White people account for the largest share of unvaccinated people, they have a higher vaccination rate than AAs, which leaves AAs at increased risk of infection, sickness, and death. Low vaccination rates have raised concerns among public health and government officials as they are likely to widen the already persistent health disparities. Therefore, it is imperative to assess $\mathrm{VH}$ and its predictors locally to strategize and improve vaccine uptake going forward. The study aims to provide a better understanding of the underlying causes of (1) VH in faithbased AAs, (2) the role of health and wellness programs in promoting COVID-19 vaccination, and (3) the knowledge gained can inform the design of context-based and culturally sensitive interventions necessary to address vaccine hesitancy. Here, we discuss perceptions on COVID vaccination of AAs involved in a faith-based wellness program following the SAGE WG Matrix of Determinants of VH.

The themes in the Matrix of Determinants of VH that influenced behaviors fell within the contextual influences category. Within this category, historical influences were a major predictor of $\mathrm{VH}$. Continued acts of violence by police against AAs, particularly during the pandemic [George Floyd: May 25, 2020, Breonna Taylor: March 13, 2020], were a significant source of distrust and tension between AAs and the government. Given the disproportionately higher rates of COVID-19-related infection and deaths, AAs were experiencing, compounded by historical injustices - poor education, housing, working environment, lack of access to preventative and medical care, and police brutality - worsened already poor levels of trust in government among AAs. The lack of trust may spread into other government efforts, like vaccinations. Findings underscore how historical experiences of inequities and contemporary movements for racial justice interact with vaccine hesitancy.

Comments from participants showed that "the past is so present." Deeply rooted injustices (e.g., racism and discrimination) impact AAs in ways that transverse all aspects of their lives (e.g., poor housing, education, and access to health), and their persistent occurrence (e.g., White police brutality against AAs) is a concern for us all. As long as the rate of fatal police shootings among AAs remains much higher than other minority groups, a lot of relationship and trust-building between government and AA citizenry still need to happen. Research has shown, time and again, that when communities trust their police, they are more likely to obey the law [53] and trust the government. Once earned, that trust can influence other decisions AAs make about their health and well-being.

Further, the striking parallel was drawn by participants between the "Never Forget" 9/11 campaign and the expectation society has on AAs to "forget" about previous unethical healthcare research in Black populations, provides critical insights into the need for restoration of trust as the foundational step towards improving vaccination rates among AAs. First, cherry-picking practices in which the government anticipates citizenry not to forget the sacrifices made by Americans for Americans during (e.g., 9/11) yet expects the same citizenry to forget how AA lives were sacrificed during the unethical practices committed on them by White Americans are wrong in themselves. Second, instead of mandating vaccines, AAs prefer to experience significant change from the government regarding how they are treated through the life course regarding access to education, healthcare, housing, and economic opportunities. The inequities experienced by African Americans are believed to stem from systemic racism, which has economically and socially disadvantaged AA for centuries resulting in a greater prevalence of underlying health conditions, such as hypertension, diabetes, obesity, and increased risk for infection and death from infectious diseases such as COVID-19 [4, 54-56]. Measures such as mandatory vaccination policies by employers, particularly when specific professions that employ primarily Black people are targeted (e.g., healthcare, hospitality, food 
industry, and travel), can be viewed as the excessive hand of government to continue to oppress minorities and thereby increase distrust.

Within the contextual influences category, "perception of pharmaceutical industry" and "communication and media environment" also influence vaccine hesitancy. Given that pharmaceutical companies work with government agencies (e.g., FDA), this observation was not surprising as participants feared that pharmaceutical companies could be in cahoots with the government to profit off AA's life. It was striking that governments failed to dilute these fears through effective and targeted communication. For AAs, trying to make an informed decision at the intersection of distrust in government, the evocation of past experiences, the Janssen vaccine scare, and the COVID-19 infodemic was complex and created worry and fear. In particular, the excess information made it difficult for people to access reliable information when they needed it [23]. The creation of uncertainty nurtures worry and fear - both of which can independently or collectively cause vaccine hesitancy [23].

Observations we made under the two categories of individual and group influences and vaccine-specific issues support research that identifies concerns for intending not to receive the COVID-19 vaccine as including knowledge/ awareness of vaccine, perception of healthcare providers, and worries about the newness and safety of the vaccine as well as about potential side effects [2, 3, 5, 6, 9]. While the infodemic discussed earlier exacerbated the already poor health literacy among AAs, some individuals' negative experience with care providers validated their lack of trust in the health system-worsening vaccine hesitancy. The question one of the participants raised, "Had I been a White person would I have been sent home the first time with my medical condition" points to a persistent issue in our healthcare system that needs to be addressed. Creating an equitable healthcare system will take intentional efforts by White healthcare providers, on the one hand, to elevate the quality of care they provide to minorities, and by AAs, on the other hand, to speak up in the face of what feels unjust. Given this paper's overarching theme, it would appear that "the past is so present." White healthcare providers need to consider their privilege compared to their minority patients. Minority and low-income patients may live in communities that lack resources (i.e., education, healthcare, and housing) to help them achieve their dreams.

Finally, evidence from the study suggests that faith-based health and wellness programs rooted in AA communities have the potential to provide trustworthy information and advocate for extra resources, such as vaccination clinics in these resource-limited communities that are home to most AA people. Because of their local presence, these programs have a contextual advantage (e.g., culture, beliefs, and values) - they have a nuanced understanding of attitudes towards and experiences of vaccines and factors influencing COVID-19 VH - all of which can help with tailoring public health messaging accordingly. Additionally, insights from this study highlight the potential value of programs founded on partnerships between local government, church leaders, and community members in promoting health. Governmentcommunity partnerships can increase the community's capacity to shape outcomes by placing community members at the center of co-creating solutions to community issues.

\section{Conclusion and Recommendations}

Although this study is based on a sample of African Americans involved in a faith-based health and wellness program in the Midwest, it underscores crucial issues that the US needs to address aggressively. First, the abrasive relationship between the AA community and the police must be addressed. There is a need for aggressive efforts to train and provide technical assistance for police officers who engage with minoritized residents to repair damaged police-community relations. Second, the federal government should maximize communication and collaboration between AAs, government, and healthcare providers. There is a need to improve transparency in basic information about how vaccines are developed and work, their effectiveness, and related side and long-term effects. Government should embrace AA communities as trusted and invested partners in fighting the pandemic. For example, co-creating public health messaging with key constituencies can help boost trust and vaccine uptake. Programs such as LWBF and other community organizations that have a local presence in AA communities and are trusted by residents should be funded to expand their work, to restore and strengthen community trust in government, and ultimately to impact health behaviors. Third is the lack of sustainable infrastructure for health equity and justice in AA communities. Local and state governments should engage AA communities (e.g., creating Black Leadership Advisory Boards) to advocate for formulating programs and policies that elevate AA communities $[9,41]$. Fourth, given the role race and religion play in $\mathrm{VH}$, research investigating the intersection of race and religion is needed. This research can inform the designing of vaccination programs and promote the building of collaborations between key stakeholders such as the government, faith leaders, and minority populations. Finally, given the high mutation and transmission rates of COVID-19 variants, and the development of booster vaccines, research that examines perceptions of AAs on booster shot can contribute new knowledge on vaccine hesitancy.

Acknowledgements The support we got from church leaders, lifestyle coaches, and program participants is greatly appreciated. Authors are 
also thankful for the role community partners played in recruiting participants.

Data Availability Data collected for this are available from the corresponding author [WM] on reasonable request.

\section{Declarations}

Ethics Approval Ethics approval was granted by the institutional review board at the corresponding author's university [IRB \#2052482].

Conflict of Interest The authors declare no competing interests.

\section{References}

1. Ndugga, N., et al. Latest data on COVID-19 vaccinations by race/ ethnicity. 2021 [cited 2021 October 27]; Available from: https:// www.kff.org/coronavirus-covid-19/issue-brief/latest-data-oncovid-19-vaccinations-by-race-ethnicity/.

2. Murphy, R., K. Thakar, and M. Mayhew. Coronavirus and the social impacts on Great Britain: 10 September 2021. 2021 [cited 2021 September 15]; Available from: https://www.ons.gov.uk/ peoplepopulationandcommunity/healthandsocialcare/healthandw ellbeing/bulletins/coronavirusandthesocialimpactsongreatbritain/ latest.

3. Paul E, Steptoe A, Fancourt D. Attitudes towards vaccines and intention to vaccinate against COVID-19: implications for public health communications. The Lancet Regional Health-Europe. 2021;1:100012.

4. Razai, M.S., et al., Mitigating ethnic disparities in COVID-19 and beyond. bmj, 2021. 372.

5. Robertson E, et al. Predictors of COVID-19 vaccine hesitancy in the UK household longitudinal study. Brain Behav Immun. 2021;94:41-50.

6. Laurencin CT. Addressing justified vaccine hesitancy in the Black community. J Racial Ethn Health Disparities. 2021;8(3):543-6.

7. Mills M, et al. COVID-19 vaccine deployment: behaviour, ethics, misinformation and policy strategies. London: The Royal Society \& The British Academy; 2020.

8. Miko D, et al. Qualitative assessment of vaccine hesitancy in Romania. Medicina. 2019;55(6):282.

9. Strully KW, et al. Strategies to address COVID-19 vaccine hesitancy and mitigate health disparities in minority populations. Front Public Health. 2021;9:384.

10. Nazroo J, Becares L. Evidence for ethnic inequalities in mortality related to COVID-19 infections: findings from an ecological analysis of England. BMJ open. 2020;10(12):e041750.

11. Bhala N, et al. Sharpening the global focus on ethnicity and race in the time of COVID-19. The Lancet. 2020;395(10238):1673-6.

12. Valeriani, G., et al. Addressing healthcare gaps in sweden during the COVID-19 outbreak: on community outreach and empowering ethnic minority groups in a digitalized context. in Healthcare. Multidisciplinary Digital Publishing Institute..2020

13. Center for Disease Control. Provisional death counts for coronavirus disease 2019 (COVID-19). 2021 [cited 2021 September 1]; Available from: https://www.cdc.gov/nchs/nvss/vsrr/covid_ weekly/index.htm.

14. Center for Disease Control. Demographic trends of COVID-19 cases and deaths in the US reported to CDC. 2021 [cited 2021 September 10]; Available from: https://covid.cdc.gov/covid-datatracker/\#demographics.
15. Shiels, M.S., et al., Racial and ethnic disparities in excess deaths during the COVID-19 pandemic, March to December 2020. Annals of internal medicine, 2021.

16. Ndugga, N., L. Hill, and S. Artiga. Latest data on COVID-19 vaccinations by race/ethnicity. 2021 [cited 2021 September 15]; Available from: https://www.kff.org/coronavirus-covid19/issue-brief/latest-data-on-covid-19-vaccinations-race-ethni city/.

17. O'Brien, R., The Oxford handbook of the social science of poverty. SAGE Publications Sage CA: Los Angeles, CA. 2018

18. Soares $\mathrm{P}$, et al. Factors associated with COVID-19 vaccine hesitancy. Vaccines. 2021;9(3):300.

19. MacDonald NE. Vaccine hesitancy: definition, scope and determinants. Vaccine. 2015;33(34):4161-4.

20. Moore JX, et al. Correlates of COVID-19 vaccine hesitancy among a community sample of African Americans living in the Southern United States. Vaccines. 2021;9(8):879.

21 Edwards B, et al. COVID-19 vaccine hesitancy and resistance: correlates in a nationally representative longitudinal survey of the Australian population. PloS one. 2021;16(3):e0248892.

22. Hamel Liz, et al. KFF COVID-19 vaccine monitor: April 2021. 2021 [cited 2021 July 8]; Available from: https://www.kff.org/ coronavirus-covid-19/poll-finding/kff-covid-19-vaccine-monit or-april-2021/.

23. Wiysonge, C.S., et al., Vaccine hesitancy in the era of COVID-19: could lessons from the past help in divining the future? Human vaccines \& immunotherapeutics, 2021: 1-3.

24. Hooper, M.W., A.M. Nápoles, and E.J. Pérez-Stable, No populations left behind: vaccine hesitancy and equitable diffusion of effective COVID-19 vaccines. Journal of General Internal Medicine, 2021: 1-4.

25. Betsch, C., et al., Sample study protocol for adapting and translating the 5C scale to assess the psychological antecedents of vaccination. BMJ open, 2020. 10(3): p. e034869.

26 Betsch C, et al. Beyond confidence: development of a measure assessing the $5 \mathrm{C}$ psychological antecedents of vaccination. PloS one. 2018;13(12):e0208601.

27. Larson HJ, et al. Measuring trust in vaccination: a systematic review. Hum Vaccin Immunother. 2018;14(7):1599-609.

28. Machingaidze S, Wiysonge CS. Understanding COVID-19 vaccine hesitancy. Nat Med. 2021;27(8):1338-9.

29. Kirzinger Ashley, et al. KFF COVID-19 vaccine monitor: July 2021. 2021 [cited 2021 August 15]; Available from: https://www. kff.org/coronavirus-covid-19/poll-finding/kff-covid-19-vaccinemonitor-july-2021/.

30. Khubchandani J, et al. COVID-19 vaccination hesitancy in the United States: a rapid national assessment. J Community Health. 2021;46(2):270-7.

31. Missouri Department of Health and Senior Services. COVID-19 vaccinations in Missouri. 2021 [cited 2021 October 26]; Available from: https://covidvaccine.mo.gov/data/.

32. Habersaat $\mathrm{KB}$, et al. Ten considerations for effectively managing the COVID-19 transition. Nat Hum Behav. 2020;4(7):677-87.

33. Enwezor $\mathrm{CH}$, et al. Changing attitudes toward the COVID-19 vaccine among North Carolina participants in the COVID-19 community research partnership. Vaccines. 2021;9(8):916.

34 Saluja S, et al. Disparities in COVID-19 vaccine hesitancy among Los Angeles County adults after vaccine authorization. Preventive Medicine Reports. 2021;24:101544.

35. Sharma, M., K. Batra, and R. Batra. A theory-based analysis of COVID-19 vaccine hesitancy among African Americans in the United States: a recent evidence. in Healthcare. Multidisciplinary Digital Publishing Institute. 2021

36. Quinn SC, et al. Breaking down the monolith: understanding flu vaccine uptake among African Americans. SSM-population health. 2018;4:25-36. 
37. Wismans A, et al. Psychological characteristics and the mediating role of the $5 \mathrm{C}$ model in explaining students' COVID-19 vaccination intention. PloS One. 2021;16(8):e0255382.

38. Jacobi CJ, Vaidyanathan B. Racial differences in anticipated COVID-19 vaccine acceptance among religious populations in the US. Vaccine. 2021;39(43):6351-5.

39. Olagoke AA, Olagoke OO, Hughes AM. Intention to vaccinate against the novel 2019 coronavirus disease: the role of health locus of control and religiosity. J Relig Health. 2021;60(1):65-80.

40. Corcoran KE, Scheitle CP, DiGregorio BD. Christian nationalism and COVID-19 vaccine hesitancy and uptake. Vaccine. 2021;39(45):6614-21.

41. Privor-Dumm L, King T. Community-based strategies to engage pastors can help address vaccine hesitancy and health disparities in Black communities. J Health Commun. 2020;25(10):827-30.

42. Salazar, L.F., R.A. Crosby, and R.J. DiClemente, Research methods in health promotion. John Wiley \& Sons. 2015

43. Creswell, J.W. and J.D. Creswell, Research design: qualitative, quantitative, and mixed methods approaches. Sage publications. 2017

44. Korstjens, I. and A. Moser, Series: practical guidance to qualitative research. Part 2: context, research questions and designs. Eur J Gen Pract 2017 23(1): 274-279.

45. Saunders B, et al. Saturation in qualitative research: exploring its conceptualization and operationalization. Qual Quant. 2018;52(4):1893-907.

46. Fereday J, Muir-Cochrane E. Demonstrating rigor using thematic analysis: a hybrid approach of inductive and deductive coding and theme development. Int J Qual Methods. 2006;5(1):80-92.
47. Braun, V. and V. Clarke, What can "thematic analysis" offer health and wellbeing researchers? Taylor \& Francis. 2014

48. Hill CE, et al. Consensual qualitative research: an update. J Couns Psychol. 2005;52(2):196.

49. Cooper, H.E., et al., APA handbook of research methods in psychology, Vol 1: foundations, planning, measures, and psychometrics. American Psychological Association. 2012

50. Singh J. Critical appraisal skills programme. J Pharmacol Pharmacother. 2013;4(1):76.

51. Vonderlehr RA, et al. Untreated syphilis in the male Negro: a comparative study of treated and untreated cases. J Am Med Assoc. 1936;107(11):856-60.

52. Wouters, O., et al., Challenges in ensuring global access to COVID-19 vaccines: production, affordability, allocation, and deployment. Lancet [Internet]. 2021, Elsevier.

53. La Vigne N, Jannetta J, Fontaine J, Lawrence DS, Esthappan S. The national initiative for building community trust and justice. Washington, DC: Urban Institute; 2019. p. 1-13.

54. Bogart, L.M., et al., COVID-19 related medical mistrust, health impacts, and potential vaccine hesitancy among Black Americans living with HIV. J Acquired Immune Deficiency Syndromes (1999), 2021. 86(2): 200

55. Williams DR, Lawrence JA, Davis BA. Racism and health: evidence and needed research. Annu Rev Public Health. 2019;40:105-25.

56 Paradies Y, et al. Racism as a determinant of health: a systematic review and meta-analysis. PloS One. 2015;10(9):e0138511.

Publisher's Note Springer Nature remains neutral with regard to jurisdictional claims in published maps and institutional affiliations. 